\title{
The East Asian Regional Office of Astronomy for Development
}

\author{
Richard de Grijs ${ }^{1}$, Ziping Zhang ${ }^{2}$ and Jinhua $\mathbf{H e}^{3}$ \\ ${ }^{1}$ Kavli Institute for Astronomy \& Astrophysics, Peking University, Yi He Yuan Lu 5, Hai Dian \\ District, Beijing 100871, China \\ email: grijs@pku.edu.cn \\ ${ }^{2}$ Beijing Planetarium, 138 Xizhimenwai Dajie, Xicheng District, Beijing 100044, China \\ ${ }^{3}$ Yunnan Observatories, Chinese Academy of Sciences, Phoenix Mountain, East District, \\ Kunming 650011, China
}

\begin{abstract}
At the 2012 General Assembly of the International Astronomical Union (IAU), the Office of Astronomy for Development announced a number of exciting new partnerships to assist with the IAU's decadal strategic plan (2010-2020). These landmark decisions included establishing a new coordinating centre that aims at using astronomy as a tool for development in East Asia. The agreement covers two important functions. One is known as a Regional Node, which entails the coordination of astronomy-for-development activities in countries within the general geographical region of East Asia. The other is known as a Language Expertise Centre which deals with all aspects relating to (mainly) the Chinese language and culture. The impact of the latter may obviously spread well beyond the geographical region to other parts of the world. Here we provide an update of the achievements and aims of the East Asian Office of Astronomy for Development.
\end{abstract}

Keywords. miscellaneous, sociology of astronomy

\section{Facilitation of Astronomy for Development in the far East}

An important component of the International Astronomical Union's (IAU) Strategic Plan 2010-2020 is the adoption of a "bottom-up" approach, with a substantial degree of decentralisation. This involves the appointment of regional development coordinators and the designation of regional nodes. The first of these pioneering agreements, concerning a coordinating centre established in the East Asian region (in China), was signed on Tuesday, 21 August, 2012. The main institutes involved in the East Asian Regional Office of Astronomy for Development (EA-ROAD) are the Kavli Institute for Astronomy and Astrophysics (KIAA, Peking University) in Beijing, Beijing Planetarium and Yunnan Observatories, Chinese Academy of Sciences (YNAO). Our institutes are supported in our efforts by a number of important partners, including the National Astronomical Observatories of the Chinese Academy of Sciences (NAOC), the East Asian Observatory (EAO), the National University of Mongolia (NUM) and Pyongyang Astronomical Observatory (PAO).

Our key geographical focus area of interest comprises mainland China, Mongolia and the Democratic People's Republic of Korea (DPRK). Through the EAO, we have indirect links to Japan (and the IAU Office of Public Outreach at the National Astronomical Observatories of Japan; NAOJ), Taiwan (Academia Sinica Institute of Astronomy and Astrophysics), and the Republic of Korea (Korean Astronomy and Space Science Institute). This area covers of order a quarter of the world's population, and given the small size of the EA-ROAD's steering committee, our role is mainly one of facilitation and 
information exchange rather than that of a major driver of practical implementation. Nevertheless, we do not consider the boundaries to our main focus area to imply exclusion of activities in neighbouring countries if and when the opportunity arises to make a difference there in the context of our "Astronomy for Development" remit.

The majority of EA-ROAD facilitation and active engagement during the first triennium of its operation occurred in China. Astronomy in China is concentrated in a number of large institutions under the umbrella of the Chinese Academy of Sciences $(\mathrm{CAS})$ and in a few university departments (predominantly in Beijing, Nanjing, and Xiamen). CAS institutes include, among others, NAOC, YNAO, Shanghai Astronomical Observatory (SHAO), Purple Mountain Observatory (Nanjing), the Nanjing Institute of Astronomical Optical Technology, and Xinjiang Astronomical Observatory. We have invited representatives from SHAO and NUM to join the EA-ROAD's steering committee. We have also established contacts with the team running the new FAST radio telescope, under development in Guizhou province, aiming at setting up a teacher training event in collaboration with a number of tertiary education colleges we incorporated as associated partners, thanks to significant efforts by our YNAO-based steering committee member.

Despite this focus on China, we have also engaged positively with our colleagues in both Mongolia and the DPRK. We organised a summer school and the first ever international conference aimed at professional astrophysicists at the NUM in September 2014. Although Mongolian astronomy dates back thousands of years, the country has only been an interim member of the IAU since 2006. The small Mongolian astronomical community has interacted with foreign organisations through several workshops and schools, most prominently the 2008 Astronomical Summer School, held in Ulaanbaatar, and also through interaction with NAOJ. The Mongolian astronomical community is keen to develop their research abilities and exposure to attain an internationally competitive level.

The long-term aims of the DPRK astronomical community are to increase the level of astronomy in all areas of research, education and public outreach to an internationally acceptable high level. This includes conducting cutting-edge professional research and astronomy education of the general public to a sufficient level to secure the benefit of astronomy as a tool of national capacity building. To support this aim, we hosted several researchers from PAO for 6 months each at NAOC's Huairou Solar Observatory near Beijing. In addition, two of their colleagues spent 6 months at Leiden University (Netherlands) thanks to the great efforts of Prof. George Miley.

We have also facilitated Master's and PhD student exchanges with Mongolia, Nepal, Indonesia, Bangladesh and other regional centres. In addition, we have actively worked on establishing links with external partners, including the UK's Institute of Physics, with whom we support monthly science days in underresourced schools for children from so-called migrant families in Beijing, and with the European Union's EURAXESS networking organisation, bringing together Chinese and international researchers.

The EA-ROAD team is very keen to involve a larger fraction of the astronomical community in the wider region. For more details of our achievements and ambitions, we refer the reader to the EA-ROAD website, http://eastasia.astro4dev.org. A full description of the EA-ROAD, in Chinese, and a call for participation was published in The Amateur Astronomer (published by Beijing Planetarium; also read by many professional astronomers around the country) of October 2012 (pp. 40-43); an English version is available at http://astro-expat.info/ROAD-EastAsia.pdf. Further details can be obtained by email, eastasia@astro4dev.org or chinese@astro4dev.org (Chinese or English). We will redouble our efforts to engage the community during the annual meeting of the Chinese Astronomical Society, held on the campus of Peking University in October 2015. 